\title{
Dose matters for stereotactic body radiotherapy for early stage non-small cell lung cancer
}

\author{
Christian C. Okoye ${ }^{1}$, C. Jane Cho ${ }^{2}$, Mitchell Liu ${ }^{3}$, Alexander V. Louie ${ }^{4}$, Olusola Obayomi-Davies ${ }^{5}$, \\ Shankar Siva ${ }^{6}$, Simon S. Lo ${ }^{2}$
}

${ }^{1}$ Department of Radiation Oncology, St. Bernards Medical Center, Jonesboro, AR, USA; ${ }^{2}$ Department of Radiation Oncology, University of Washington School of Medicine, Seattle, WA, USA; ${ }^{3}$ BC Cancer-Vancouver Clinic, Vancouver, Canada; ${ }^{4}$ Department of Radiation Oncology, Sunnybrook Health Science Centre, Toronto, ON, Canada; ${ }^{5}$ Department of Radiation Oncology, WellStar Medical Group, Marietta, GA, USA; ${ }^{6}$ Sir Peter MacCallum Department of Oncology, University of Melbourne, Melbourne, Australia

Correspondence to: Simon S. Lo, MB, ChB, FACR, FASTRO. Professor \& Vice Chair for Strategic Planning, Director of Stereotactic Body Radiation Therapy, Professor of Neurological Surgery, UW School of Medicine, Department of Radiation Oncology, 1959 NE Pacific St., Seattle, WA 98195 , USA. Email: simonslo@uw.edu.

Comment on: Moreno AC, Fellman B, Hobbs BP, et al. Biologically Effective Dose in Stereotactic Body Radiotherapy and Survival for Patients With Early-Stage NSCLC. J Thorac Oncol 2020;15:101-9.

Submitted Apr 06, 2020. Accepted for publication Apr 17, 2020.

doi: 10.21037/atm-20-3149

View this article at: http://dx.doi.org/10.21037/atm-20-3149

For patients with early-stage, node-negative non-small cell lung cancer (NSCLC), stereotactic body radiation therapy (SBRT) has been a welcome addition as a noninvasive, curative treatment option for appropriately selected patients. It has evolved from an abundance of literature demonstrating higher tumor control with higher biologically equivalent doses (BED) of radiation along with improvements in patient evaluation and staging, patient setup and motion management, treatment planning, image guidance, radiation delivery, and understanding of relevant, dose-related toxicities. However, the dose-fractionation schemes of SBRT for early-stage NSCLC remain loosely defined and are based largely on toxicity, with the implicit assumption that all regimens are similarly effective.

In the seminal paper by Onishi et al., patients with stage I NSCLC treated with various SBRT schedules were found to have lower rates of locoregional recurrence and improved overall survival (OS) with BED of greater than $100 \mathrm{~Gy}_{10}(1)$. Additional studies have confirmed these results and established the superiority of doses greater than $100 \mathrm{~Gy}_{10}$ and attempts at curing patients with early-stage NSCLC aim to achieve these doses. While similar $\mathrm{BED}_{10}$ doses can be achieved over more than 5 fractions (e.g., 60 Gy in 8 fractions), as is commonly done outside of the United States, recent ASTRO guidelines consider these regimens as alternatives to treatment schedules with 5 or fewer fractions (2). What remains unclear is whether calculating a $\mathrm{BED}_{10}$ is an accurate method for stratifying the efficacy of SBRT regimens (3), and if so, whether there are any significant differences in outcomes associated with $B E D_{10}$.

Moreno et al. attempt to address the latter question in their recent publication (4). Specifically, the authors used the National Cancer Database (NCDB) to identify patients treated with SBRT as a curative intent modality for a clinical stage I (T1-2aN0 per AJCC $7^{\text {th }}$ edition) NSCLC to investigate whether a higher $\mathrm{BED}_{10}$ is associated with improved survival outcomes. They were unable to directly account for tumor centrality, a known confounder, but attempted to account for this by limiting cohort to those receiving at least $100 \mathrm{~Gy}_{10}$. Initially, an exploratory analysis was used to determine the appropriate $\mathrm{BED}_{10}$ cutoff relative to OS; subsequently, patients were dichotomized by $\mathrm{BED}_{10}$, with low BED corresponding to $100-129 \mathrm{~Gy}_{10}$ and high BED corresponding to doses $130 \mathrm{~Gy}_{10}$ and above. The cohort was further refined through 1:1 propensity scorematching, with $\mathrm{BED}_{10}$ stratification and age at diagnosis, comorbidity, tumor size, tumor histology, and tumor location as covariates.

With a median follow up of 26 months, 5-year OS rates between propensity-matched high and low BED 
cohorts were $34 \%$ and $26 \%(\mathrm{P}=0.039)$, respectively, and on multivariate analysis, receipt of low BED was associated with significantly worse survival (HR $1.046, \mathrm{P}=0.032$ ). Low BED regimens became more common over the study period corresponding with key publications in the literature. The authors concluded that high BED regimens may confer a survival benefit, low BED regimens have become more common clinically, and additional studies are needed to determine the dose-response and associated toxicities of high BED regimens.

This work provides valuable insight into the role of SBRT in clinical practice and extend the work of Koshy et al. in a previous NCDB analysis (5). In that study, 498 patients with T1-2N0 NSCLC treated with SBRT from an earlier era (2003-2006) were similarly evaluated for the correlation between $\mathrm{BED}_{10}$ (dichotomized at 150 Gy $\mathrm{BED}_{10}$ ) and OS. Through their analysis, a significant interaction was detected between T-stage and $\mathrm{BED}_{10}$, where the 3-year OS was no different among patients with T1 tumors while patients with $\mathrm{T} 2$ tumors saw a significant increase in their 3 -year OS with high versus low BED treatments (37\% vs. $24 \%, \mathrm{P}=0.01)$. These differences among patients with $\mathrm{T} 2$ tumors persisted in multivariate analysis.

While many of the limitations in this analysis are inherent in large databases like the NCDB, strengths of this updated analysis include the very large and modern sample size that reflects current patterns of practice with propensity-matching across known and available potential confounders between treatment strategies (though differences persisted in race and facility type). The analysis also limited the patient cohort to what we would consider standard SBRT treatments in the US, including those who received $\mathrm{BED}_{10}>100$ Gy over three to five fractions. The primary comparison between high and low BED regimens was informed by an initial exploratory analysis of the same cohort, limiting the generalizability of the findings.

Throughout the study period, radiation target dose prescriptions and calculation methods have changed significantly. In the early 2000s, tumor doses were more often prescribed to point(s) within the target structure without heterogeneity corrections, while now, doses are almost universally prescribed to the target periphery with heterogeneity corrections. In fact, because dose-volume histogram (DVH) characteristics are evaluated for all critical structures, heterogeneity across the entire tumor volume may be used intentionally to allow for lower doses in areas immediately adjacent to organs at risks (OARs). However, the lower peripheral doses to the planning target volume
(PTV) and OARs within the PTV may not be accurately captured by the prescription dose alone and the impact of marginal dose and/or tumor heterogeneity on outcomes remains incompletely understood. In summary, the lack of dosimetric data within the NCDB limits the reliability of the major dependent variable, $\mathrm{BED}_{10}$, as the nominal prescription dose may not have the same meaning across different prescribing physicians, given the aforementioned changes in practice pattern. Prospective studies mitigate these effects by creating a homogeneous set of parameters for which the outcomes are based, while future cohort studies may instead use DVH-based dose reporting. The use of robust dose calculation methods and quality heterogeneity correction are also required to understand the actual spatial dose distribution and its effect on tumor control (6).

Several studies have evaluated the authors' primary conclusion that higher $\mathrm{BED}_{10}$ doses may correlate with improved OS at doses above $100 \mathrm{~Gy}_{10}$. A meta-analysis performed by Zhang et al. in 2011 showed concern for higher doses $\left(>146 \mathrm{~Gy}_{10}\right)$ causing lower survival compared to medium (83.2-106 $\left.\mathrm{Gy}_{10}\right)$ and medium to high (106-146 $\left.\mathrm{Gy}_{10}\right)$ doses, leading authors to conclude that medium and medium to high doses may be most appropriate (7). Mak et al. reached a similar conclusion after reviewing their institutional experience of 75 patients treated with SBRT for early-stage lung cancer, finding no significant impact of $\mathrm{BED}_{10} \geq 151.2$ (HR 0.88, $\mathrm{P}=0.76$ ) on freedom from any recurrence in univariate analysis (8). Ma et al. compared 3 fraction (dose, median: 60 Gy or $151 \mathrm{~Gy}_{10}$ ) and 5 fraction (dose, median: 50 Gy or $94 \mathrm{~Gy}_{10}$ ) regimens, and in a propensity score-matched cohort of 94 patients, no differences were observed in OS $(\mathrm{P}=0.33)$ or local failure $(\mathrm{P}=0.86)$ (9). In contrast, Kestin et al. reviewed a multi-institutional experience with T1-2N0 NSCLC, and determined an optimal $\mathrm{BED}_{10} \mathrm{PTVmean}$ cutpoint for local recurrence to be $125 \mathrm{~Gy}$, with a corresponding 2 -year local recurrence of $4 \%$ vs. $17 \%$ for PTVmean $>$ $v s .<125$ Gy, respectively (10). Additionally, Zhao et al. reviewed their institutional experience with T1-2N0M0 NSCLC undergoing hypofractionated RT (70 Gy over 10 fractions) and SABR (50 Gy over 4 fractions). Cutoff values determined by receiver operating characteristic (ROC) curve analysis relating to local and/or lobar recurrence included the minimum $\mathrm{BED}_{10}$ to $95 \%$ of the PTV >86 Gy and a highly correlated PTVmean BED ${ }_{10}$ $>130$ Gy (significant on univariate analysis; corresponds to a PTVmean physical dose of 55 Gy over 4 fractions) (11). 
Finally, Stephans et al. reported on patients with peripheral tumors receiving various high and low BED regimens, demonstrating reduced 2-year local failure rate with a high BED regimen (60 Gy in 3 fractions, $4.3 \%$; 30-34 Gy in 1 fraction, $21 \%$; 48-50 Gy in $4-5$ fractions, $15.5 \%$; $50-60$ Gy in 8-10 fractions, $13.3 \%$ ) without differences in nodal failure, distant failure, or OS (12).

The main drawbacks of these studies are that they are retrospective, underpowered, inclusive of BED doses below recommended limit (i.e., $\mathrm{BED}_{10}<100 \mathrm{~Gy}$ ), and limited capacity to account for known prognostic factors that impact tumor control and survival. The studies are also difficult to compare and apply in practice due to differences in what constitutes high and low BED regimens (with cutoffs ranging from $125 \mathrm{~Gy}_{10}$ to $150 \mathrm{~Gy}_{10}$ ) and limited validation in independent datasets. While the data remains mixed and potentially complicated by other factors (such as tumor size, as stated below), the current study lends support toward the role for higher $\mathrm{BED}_{10}$ doses in treating earlystage NSCLC, though the prospective evaluation of precise DVH metrics remains necessary to ensure the safety of such an approach.

In studies that evaluate tumor size, many confirm a significant correlation between tumor dose and tumor control that is affected by tumor size. In a registry study of early-stage lung cancer patients treated with SBRT reported by Davis et al., prescription $\mathrm{BED}_{10}$ of $<105$, 105-149, and >150 Gy among T2 patients resulted in significant improvements in 17 -month local control (LC) of $43 \%, 74 \%$, and $95 \%$, respectively; no dose-response was seen in T1 tumors (13). Koshy et al. also noted a significant interaction between T-stage and $\mathrm{BED}_{10}$ in their sensitivity analysis, limiting the cohort to patients receiving a $\mathrm{BED}_{10}$ $>100 \mathrm{~Gy}$ (5). Using a cutoff of $150 \mathrm{~Gy}_{10}$, the 3-year OS among patients with $\mathrm{T} 2$ tumors was significantly improved with $\mathrm{BED}_{10}>150$ Gy (37\% vs. $17 \%$, HR 0.32, $\mathrm{P}<0.0001$ ), but not among $\mathrm{T} 1$ tumors $(\mathrm{P}=0.952)$. Moving forward, given that tumor size likely correlates with LC and OS in NSCLC patients who receive SBRT, it should be used to stratify patients for planned subset analysis in prospective studies that evaluate different SBRT regimens. In contrast, lower $\mathrm{BED}_{10}$ regimens may be appropriate in treating smaller tumors as part of a risk-based approach, with similar efficacy (14). Other settings that may be appropriate for lower dosing include those immediately adjacent to critical OARs and patients with comorbidities or advanced age who may not tolerate the added risk of toxicity (15).

Whether higher doses lead to increased toxicity likely also depends on factors unaccounted for in this analysis, including tumor centrality and individual OAR dose constraints. The report from Timmerman et al. outlined the concern for toxicity among centrally located tumors (16), leading to the adoption of various protracted and/or lower BED regimens attempting to limit toxicity. Recently, Bezjak et al. published outcomes from RTOG 0813, the phase I-II dose-escalation study for central tumors, which confirmed limited toxicity when adhering to strict dose constraints (17). While this may be reasonable for most patients, questions remain about the best dose and fractionation schedule that maximizes LC and minimizes toxicity for patients with tumors that are ultracentral or intimately associated (i.e., GTV or PTV overlap) with central OARs, particularly the proximal bronchial tree and esophagus. Some studies have shown very high rates of Grade 3-5 toxicity with ultracentral tumors, even with protracted fractionation schedules and lower $\mathrm{BED}_{10}(18)$ or high rates of local failure with tumors treated more conservatively (19). Although such trade-offs between TCP and toxicity are difficult to navigate, ongoing trials such as the HILUS trial (20) and SUNSET trial (NCT03306680) (21) may provide better information about the risks to make more informed decisions.

Could the inferior survival outcomes of the lower BED regimens be due to a higher proportion of patients with centrally located tumors? While the authors attempted to mitigate the confounding by tumor centrality by limiting the analysis to $\mathrm{T} 2 \mathrm{a}\left(\right.$ size $<5 \mathrm{~cm}$ ) lesions per AJCC $7^{\text {th }}$ edition treated with doses $>100 \mathrm{~Gy}_{10}$, it is certainly plausible that protracted fraction schedules with lower $\mathrm{BED}_{10}$ correlate with tumor centrality, as physicians attempt to reduce toxicities associated with mediastinal and hilar structures while increasing the number of patients eligible for SBRT. Central tumors are associated with an increased risk of occult nodal metastases (22), and the adverse effects of inadequate staging methods, including PET/CT and mediastinal lymph node sampling, may disproportionately affect these patients. This potential for confounding again demonstrates the importance of further prospective evaluation of the association between dose and OS.

As the SBRT data has matured, many suitable fractionation schemes have emerged. Among patients receiving $100 \mathrm{~Gy}_{10}$ or more, the authors have shown that from 2004-2014, notable changes in SBRT prescription doses have occurred, including the decline of 3 fraction regimens from its peak in 2007 and an inversely proportional rise of 50 Gy in 5 fractions (4). While these differences are 
unlikely due to changes in patient presentation, the authors attribute them to significant publications and changes in recommendations. In the absence of convincing data, groups have recommended lower BED regimens, claiming they have similar efficacy with a lower toxicity profile (23), a premise called into question by the present study. Another potential contributing factor is a patient's candidacy for SBRT. Radiation oncologists may be offering SBRT to more patients, including those previously receiving fractionated radiotherapy such as patients with central tumors.

A striking revelation in this analysis is the preference for increasingly protracted fractionation schedules since 2007 , shortly after a publication of increased toxicity with central tumors treated with high BED regimens (16). Abruptly, the global radiation oncology community is facing a unique challenge with resource constraints during the SARS COV-2 pandemic. Perhaps it is time to rethink the utilization of more severe hypofractionation schedules. While the authors did not address single-fraction schedules, perhaps due to the breakdown of the linear-quadratic model with large single doses of radiation, this strategy may be particularly attractive during the current crisis. In light of this, two randomized phase II clinical trials supporting the use of single-fraction SBRT in peripheral, early-stage NSCLC are certainly worth noting [NRG/ RTOG 0915 (NCT00960999) (24) and Roswell Park (NCT00843726) (25)], as they confirm excellent long term tumor control with low toxicity rates in appropriately selected patients.

In conclusion, while the notion of SBRT regimens needing at least $100 \mathrm{~Gy}_{10}$ for definitive treatment in early-stage NSCLC is widely accepted, the superiority of any regimen in any specific clinical scenario remains inconclusive (aside from increased toxicity of central lesions treated in 3 fractions), and yet many clinicians have moved toward lower BED treatment regimens. This work demonstrates that higher $\mathrm{BED}_{10}$ regimens are associated with and may lead to improved survival outcomes. Limitations in our understanding of radiobiologic modeling of high dose per fraction treatments may impair our ability to compare outcomes across the various treatment schedules, while currently available studies demonstrate such significant heterogeneity in reporting DVH characteristics of PTVs and OARs that detailed analyses are difficult. The available outcomes suggest that tumor size may influence the required dose to achieve tumor control, and other patient subsets, including the elderly and those with central tumors, may benefit from lower equivalent doses.
RTOG 0813 provides robust guidelines for safe delivery of SBRT for centrally located tumors but the percentage of ultracentral tumors was low. Ultimately, a regimen that will yield the most favorable therapeutic ratio for the individual patient should be chosen. Future prospective validation of these treatment characteristics, particularly related to target and OAR DVH characteristics, are needed.

\section{Acknowledgments}

Funding: None.

\section{Footnote}

Provenance and Peer Review: This article was commissioned by the editorial office, Annals of Translational Medicine. The article did not undergo external peer review.

Conflicts of Interest: All authors have completed the ICMJE uniform disclosure form (available at http://dx.doi. org/10.21037/atm-20-3149). SS reports grants from Varian Industries outside the submitted work; AVL reports personal fees from Varian Medical Systems Inc. outside the submitted work; SSL reports research support from Elekta AB in Elekta Gamma Knife ICON Expert Group outside of submitted work. The other authors have no conflicts of interest to declare.

Ethical Statement: The authors are accountable for all aspects of the work in ensuring that questions related to the accuracy or integrity of any part of the work are appropriately investigated and resolved.

Open Access Statement: This is an Open Access article distributed in accordance with the Creative Commons Attribution-NonCommercial-NoDerivs 4.0 International License (CC BY-NC-ND 4.0), which permits the noncommercial replication and distribution of the article with the strict proviso that no changes or edits are made and the original work is properly cited (including links to both the formal publication through the relevant DOI and the license). See: https://creativecommons.org/licenses/by-nc-nd/4.0/.

\section{References}

1. Onishi H, Shirato H, Nagata Y, et al. Hypofractionated Stereotactic Radiotherapy (HypoFXSRT) for Stage I Nonsmall Cell Lung Cancer: Updated Results of 257 Patients 
in a Japanese Multi-institutional Study. J Thorac Oncol 2007;2:S94-S100.

2. Videtic GMM, Donington J, Giuliani M, et al. Stereotactic body radiation therapy for early-stage non-small cell lung cancer: Executive Summary of an ASTRO Evidence-Based Guideline. Pract Radiat Oncol 2017;7:295-301.

3. Song CW, Cho LC, Yuan J, et al. Radiobiology of stereotactic body radiation therapy/stereotactic radiosurgery and the linear-quadratic model. Int J Radiat Oncol Biol Phys 2013;87:18-9.

4. Moreno AC, Fellman B, Hobbs BP, et al. Biologically Effective Dose in Stereotactic Body Radiotherapy and Survival for Patients With Early-Stage NSCLC. J Thorac Oncol 2020;15:101-9.

5. Koshy M, Malik R, Weichselbaum RR, et al. Increasing radiation therapy dose is associated with improved survival in patients undergoing stereotactic body radiation therapy for stage I non-small-cell lung cancer. Int J Radiat Oncol Biol Phys 2015;91:344-50.

6. Ohri N, Tome W, Kalnicki S, et al. Stereotactic body radiation therapy for stage I non-small cell lung cancer: The importance of treatment planning algorithm and evaluation of a tumor control probability model. Pract Radiat Oncol 2018;8:e33-9.

7. Zhang J, Yang F, Li B, et al. Which is the optimal biologically effective dose of stereotactic body radiotherapy for Stage I non-small-cell lung cancer? A meta-analysis. Int J Radiat Oncol Biol Phys 2011;81:e305-16.

8. Mak RH, Hermann G, Lewis JH, et al. Outcomes by tumor histology and KRAS mutation status after lung stereotactic body radiation therapy for early-stage nonsmall-cell lung cancer. Clin Lung Cancer 2015;16:24-32.

9. Ma SJ, Cummings M, Serra LM, et al. Three- Versus FiveFraction Regimens of Stereotactic Body Radiotherapy for Peripheral Early-Stage Non-Small-Cell Lung Cancer: A Two-Institution Propensity Score-Matched Analysis. Clin Lung Cancer 2018;19:e297-e302.

10. Kestin L, Grills I, Guckenberger M, et al. Dose-response relationship with clinical outcome for lung stereotactic body radiotherapy (SBRT) delivered via online image guidance. Radiother Oncol 2014;110:499-504.

11. Zhao L, Zhou S, Balter P, et al. Planning Target Volume D95 and Mean Dose Should Be Considered for Optimal Local Control for Stereotactic Ablative Radiation Therapy. Int J Radiat Oncol Biol Phys 2016;95:1226-35.

12. Stephans KL, Woody NM, Reddy CA, et al. Tumor Control and Toxicity for Common Stereotactic Body Radiation Therapy Dose-Fractionation Regimens in Stage
I Non-Small Cell Lung Cancer. Int J Radiat Oncol Biol Phys 2018;100:462-9.

13. Davis JN, Medbery C 3rd, Sharma S, et al. Stereotactic body radiotherapy for early-stage non-small cell lung cancer: clinical outcomes from a National Patient Registry. J Radiat Oncol 2015;4:55-63.

14. Trakul N, Chang CN, Harris J, et al. Tumor volumeadapted dosing in stereotactic ablative radiotherapy of lung tumors. Int J Radiat Oncol Biol Phys 2012;84:231-7.

15. Janssen S, Kaesmann L, Rudat V, et al. Stereotactic Body Radiotherapy (SBRT) with Lower Doses for Selected Patients with Stage I Non-small-cell Lung Cancer (NSCLC). Lung 2016;194:291-4.

16. Timmerman R, McGarry R, Yiannoutsos C, et al. Excessive toxicity when treating central tumors in a phase II study of stereotactic body radiation therapy for medically inoperable early-stage lung cancer. J Clin Oncol 2006;24:4833-9.

17. Bezjak A, Paulus R, Gaspar LE, et al. Safety and Efficacy of a Five-Fraction Stereotactic Body Radiotherapy Schedule for Centrally Located Non-Small-Cell Lung Cancer: NRG Oncology/RTOG 0813 Trial. J Clin Oncol 2019;37:1316-25.

18. Tekatli H, Haasbeek N, Dahele M, et al. Outcomes of Hypofractionated High-Dose Radiotherapy in Poor-Risk Patients with "Ultracentral" Non-Small Cell Lung Cancer. J Thorac Oncol 2016;11:1081-9.

19. Meng MB, Wang HH, Zaorsky NG, et al. Risk-adapted stereotactic body radiation therapy for central and ultracentral early-stage inoperable non-small cell lung cancer. Cancer Sci 2019;110:3553-64.

20. Lindberg K, Bergström P, Brustugun OT, et al. OA24.05 The Nordic HILUS-Trial - First Report of a Phase II Trial of SBRT of Centrally Located Lung Tumors. J Thorac Oncol 2017;12:S340.

21. Giuliani M, Mathew AS, Bahig H, et al. SUNSET: Stereotactic Radiation for Ultracentral Non-Small-Cell Lung Cancer-A Safety and Efficacy Trial. Clin Lung Cancer 2018;19:e529-32.

22. Gao SJ, Kim AW, Puchalski JT, et al. Indications for invasive mediastinal staging in patients with early nonsmall cell lung cancer staged with PET-CT. Lung Cancer 2017;109:36-41.

23. Falkson CB, Vella ET, Yu E, et al. Guideline for radiotherapy with curative intent in patients with earlystage medically inoperable non-small-cell lung cancer. Curr Oncol 2017;24:e44-9.

24. Videtic GM, Paulus R, Singh AK, et al. Long-term 
Follow-up on NRG Oncology RTOG 0915 (NCCTG N0927): A Randomized Phase 2 Study Comparing 2 Stereotactic Body Radiation Therapy Schedules for Medically Inoperable Patients With Stage I Peripheral Non-Small Cell Lung Cancer. Int J Radiat Oncol Biol Phys 2019;103:1077-84.

Cite this article as: Okoye CC, Cho CJ, Liu M, Louie AV, Obayomi-Davies O, Siva S, Lo SS. Dose matters for stereotactic body radiotherapy for early stage non-small cell lung cancer. Ann Transl Med 2020;8(18):1197. doi: 10.21037/atm-20-3149
25. Singh AK, Gomez-Suescun JA, Stephans KL, et al. One Versus Three Fractions of Stereotactic Body Radiation Therapy for Peripheral Stage I to II Non-Small Cell Lung Cancer: A Randomized, Multi-Institution, Phase 2 Trial. Int J Radiat Oncol Biol Phys 2019;105:752-9. 